\title{
Mycobacteria Degrading Polycyclic Aromatic Hydrocarbons
}

Growing interest in microorganisms capable of degrading environmentally hazardous chemicals had led to the characterization of members of the genus Mycobacterium capable of degrading volatile aliphatic hydrocarbons and polyaromatic and chlorinated hydrocarbons. Strains degrading polycyclic aromatic hydrocarbons include Mycobacterium sp. strain PYR-1 (1) (= DSM 7251), Mycobacterium sp. strain BB1 (4) (= DSM 9487), Mycobacterium sp. strain PAH 135 (5), and Mycobacterium hodleri sp. nov. (2). Stackebrandt and coworkers have recently characterized the phylogenetic position of some of the strains by $16 \mathrm{~S}$ rRNA sequencing: Mycobacterium hodleri was established as a novel species, while strain PYR-1 was found to be indistinguishable from $M$. austroafricanum and strain PAH 135 was found to be highly related to $M$. aichiense by this type of analysis $(2,3)$. We have recently determined the 16S rRNA sequence of strain BB1 (sequence accession number EMBL X81891) and found it to be nearly identical to that of $\mathrm{Myco}$ bacterium gilvum (sequence accession number X55599, homology $99.8 \%$ ). Sequence determination of the reference strain of M. gilvum ATCC 43909 revealed that the minor nucleotide differences with strain BB1 were due to sequencing errors in the previously published sequence, X55599. We conclude that strain BB-1 is in all likelihood identical to $M$. gilvum. This conclusion is supported by the mycolic acid profile (presence of mycolates I, II, and VI) and by physiological properties (kindly provided by V. Vincent, Institut Pasteur [Table 1]).

\section{REFERENCES}

1. Kelley, L., J. P. Freeman, F. E. Evans, and C. E. Cerniglia. 1993. Identification of metabolites from the degradation of fluoranthene by Mycobacterium sp. strain PYR-1. Appl. Environ. Microbiol. 59:800-806.

2. Kleespies, M., R. M. Kroppenstedt, F. A. Rainey, L. E. Webb, and E. Stackebrandt. 1996. Mycobacterium hodleri sp. nov., a new member of the fast-growing mycobacteria capable of degrading polycyclic aromatic hydrocarbons. Int. J. Syst. Bacteriol. 46:683-687.

3. Pitulle, C., M. Dorsch, J. Kazda, J. Wolters, and E. Stackebrandt. 1992. Phylogeny of rapidly growing members of the genus Mycobacterium. Int. J. Syst. Bacteriol. 42:337-343.

4. Tiem, B. A., B. Boldrien, and C. Fritsche. 1993. Degradation of phenanthrene, fluorene, fluoranthene, and pyrene by a Mycobacterium species. Appl. Environ. Microbiol. 59:1927-1930.

5. Wang, R. F., W. W. Cao, and C. E. Cerniglia. 1995. Phylogenetic analysis of polycyclic aromatic hydrocarbon-degrading mycobacteria by $16 \mathrm{~S}$ rRNA sequencing. FEMS Microbiol. Lett. 130:75-84.

E. C. Böttger
P. Kirschner
B. Springer
Medizinische Mikrobiologie
Medizinische Hochschule Hant
Konstanty-Gutschow-Str. 8
30625 Hannover
Germany
W. Zumft
Lehrstuhl für Mikrobiologie
Universität Karlsruhe
76128 Karlsruhe
Germany

TABLE 1. Physiological characteristics of Mycobacterium strain BB1

\begin{tabular}{|c|c|c|c|c|c|c|c|}
\hline Parameter $^{a}$ & $\begin{array}{l}\text { Mycobacterium } \\
\text { strain BB1 }\end{array}$ & M. parafortuitum & M. gilvum & M. austroafricanum & M. chubuense & M. obuense & M. diernhoferi \\
\hline $\mathrm{NO}_{3}$ & + & + & + & + & + & - & + \\
\hline Aryl & + & - & + & + & - & + & - \\
\hline \multicolumn{8}{|l|}{ Growth at: } \\
\hline $37^{\circ} \mathrm{C}$ & + & + & + & + & + & + & + \\
\hline $42^{\circ} \mathrm{C}$ & - & + & - & - & - & - & - \\
\hline Urease & + & + & + & + & + & + & + \\
\hline Tween & + & + & + & + & - & - & + \\
\hline \multicolumn{8}{|l|}{ Growth on: } \\
\hline PNB & + & & & + & + & + & \\
\hline Tb1 & + & + & + & + & + & + & + \\
\hline $\mathrm{NH}_{2} \mathrm{OH}$ & - & - & - & - & - & - & - \\
\hline $\mathrm{NaCl}(5 \%)$ & + & + & - & + & & & - \\
\hline Cap & - & - & - & & - & - & \\
\hline EMB & - & - & - & + & - & + & - \\
\hline MacConkey & - & - & & & & & - \\
\hline CFA & - & + & + & & & - & + \\
\hline Citrase & - & + & + & + & + & + & + \\
\hline \multicolumn{8}{|c|}{ Acid production from: } \\
\hline Fructose & + & + & & + & + & + & + \\
\hline Mannitol & + & + & + & + & - & + & + \\
\hline Inositol & + & + & + & + & - & - & + \\
\hline Trehalose & + & + & + & - & - & + & + \\
\hline
\end{tabular}

${ }^{a} \mathrm{NO}_{3}$, nitrate reductase; Aryl, arylsulfatase; Tween, Tween 80 hydrolysis at 5 days; $\mathrm{PNB}, p$-nitrobenzoase $(500 \mu \mathrm{g} / \mathrm{ml}) ; \mathrm{Tb} 1$, thiosemicarbazone $(2 \mu \mathrm{g} / \mathrm{ml}) ; \mathrm{NH}{ }_{2} \mathrm{OH}$, hydroxylamine $(250 \mu \mathrm{g} / \mathrm{ml})$; CAP, capreomycin $(40 \mu \mathrm{g} / \mathrm{ml})$; EMB, ethambutol $(2 \mu \mathrm{g} / \mathrm{ml})$; CFA, iron uptake; citrase, citrate as sole carbon source. 